\title{
Quantitative coronary computed tomography angiography for the detection of cardiac allograft vasculopathy
}

\author{
Borek Foldyna $^{1,2,3}$ (1) Marcus Sandri ${ }^{4} \cdot$ Christian Luecke $^{1} \cdot$ Jens Garbade ${ }^{5} \cdot$ Robin Gohmann $^{1} \cdot$ Jochen Hahn ${ }^{5}$. \\ Julia Fischer $^{4} \cdot$ Matthias Gutberlet ${ }^{1} \cdot$ Lukas Lehmkuhl $^{1,3}$
}

Received: 18 June 2019 /Revised: 20 November 2019 / Accepted: 19 December 2019 / Published online: 16 March 2020

(C) The Author(s) 2020

\begin{abstract}
Objectives To associate coronary wall volume and composition, derived from coronary computed tomography angiography (CTA), with cardiac allograft vasculopathy (CAV) detected on invasive coronary angiography (ICA) in heart-transplanted (HTX) patients.

Methods We included consecutive adults who received ICA and coronary CTA for evaluation of CAV $\geq 10$ months after HTX. In all coronary segments, we assessed lumen and wall volumes and segmental length, calculated volume-length ratio (VLR) (volumes indexed by segmental length; $\mathrm{mm}^{3} / \mathrm{mm}$ ), wall burden (WB) (wall/wall + lumen volumes; \%), and assessed proportions of calcified, fibrotic, fibro-fatty, and low-attenuation tissue (\%) in coronary wall. We rendered independent CTA measures associated with CAV by ICA, tested their discriminatory capacity, and assessed concordance between CTA and ICA.

Results Among 50 patients ( $84 \%$ men; $53.6 \pm 11.9$ years), we analyzed 632 coronary segments. Mean interval between HTX and CTA was $6.7 \pm 4.7$ years and between ICA and CTA $1(0-1)$ day. Segmental VLR, WB, and proportion of fibrotic tissue were independently associated with $\mathrm{CAV}(\mathrm{OR}=1.06-1.27 ; p \leq 0.002)$, reaching a high discriminatory capacity (combination of all three: $\mathrm{AUC}=0.84 ; 95 \% \mathrm{CI}, 0.75-0.90)$. Concordance between CTA and ICA was higher in advanced CAV (88\%) compared with that in none (37\%) and mild (19\%) CAV. Discordance was primarily driven by a large number of segments with coronary wall changes on CTA but without luminal stenoses on ICA $(177 / 591 ; 25 \%)$.

Conclusion CTA-derived coronary wall VLR, WB, and the proportion of fibrotic tissue are independent markers of CAV. Combination of these three parameters may aid the detection of early CAV not detected by ICA, the current standard of care.

Key Points

- Coronary CTA detects CAV in HTX patients.

- Coronary wall volume-length ratio, wall burden, and proportion of fibrotic tissue are independently associated with CAV.

- In contrast to ICA, coronary CTA may identify the early stages of CAV.
\end{abstract}

Keywords Computed tomography angiography $\cdot$ Invasive coronary angiography $\cdot$ Cardiac allograft vasculopathy $\cdot$ Heart transplantation

Matthias Gutberlet and Lukas Lehmkuhl contributed equally to this work.

Electronic supplementary material The online version of this article (https://doi.org/10.1007/s00330-019-06653-3) contains supplementary material, which is available to authorized users.

Borek Foldyna

bfoldyna@mgh.harvard.edu

1 Department of Interventional and Diagnostic Radiology, University of Leipzig - Heart Center, Struempellstrasse 39, 04289 Leipzig, Germany

2 Cardiovascular Imaging Research Center, Massachusetts General Hospital - Harvard Medical School, Boston, MA, USA
3 Clinic for Radiology, Cardiovascular Center Bad Neustadt, Bad Neustadt, Germany

4 Department of Cardiology, University of Leipzig - Heart Center, Leipzig, Germany

5 University Department for Cardiac Surgery, Leipzig Heart Center, Leipzig, Germany 


\begin{tabular}{ll}
\multicolumn{2}{l}{ Abbreviations } \\
AUC & Area under the curve \\
CAV & Coronary allograft vasculopathy \\
CHD & Coronary heart disease \\
CTA & Computed tomography angiography \\
HTX & Heart transplantation \\
HU & Hounsfield units \\
ICA & Invasive coronary angiography \\
IQR & Interquartile range \\
IVUS & Intravascular ultrasound \\
ROC & Receiver operating characteristic \\
SCCT & Society of Cardiovascular Computed Tomography \\
SD & Standard deviation \\
VLR & Volume-length ratio \\
WB & Wall burden
\end{tabular}

\section{Introduction}

Induced by immune and nonimmune triggers, cardiac allograft vasculopathy (CAV) reaches a high incidence of up to $40 \%$ in the first 5-8 years after heart transplantation (HTX) and represents the leading cause of graft failure and death among HTX patients [1]. According to recent guidelines, HTX patients should be frequently monitored by invasive coronary angiography (ICA) to detect CAV (class Ia recommendation $[2,3]$ ). However, early $\mathrm{CAV}$ is characterized by vascular fibroproliferation and diffuse coronary wall changes without coronary stenosis, unlike advanced CAV, which presents with constrictive remodeling and coronary stenosis [4-7]. While ICA examines merely the coronary lumen, it focuses on stenosis and may miss early stages of CAV which occur as changes of the coronary wall. Supplemental invasive intravascular ultrasound (IVUS) can assess the coronary wall and increase sensitivity $[3,6,8,9]$. However, IVUS is accompanied by an increased risk of severe complications (up to $4 \%$ ), including coronary spasm, acute occlusions, embolism, arrhythmia, dissection, and acute thrombosis [8].

Coronary computed tomography angiography (CTA) represents a noninvasive alternative to ICA and IVUS [10-12] and has been successfully used in numerous large trials investigating coronary heart disease (CHD) [13-16] and in smaller studies of CAV [17-19]. Still, it remains unknown whether quantitative CTA measures are associated with CAV and how the diagnosis of CAV using these measures agrees with diagnosis made by ICA.

Thus, the primary aim of this study was to determine CTAderived quantitative measures of coronary segments (i.e., volumes of the coronary lumen and wall as well as wall composition) in HTX patients and to investigate their association with CAV. Second, we aimed to determine the concordance between CTA and ICA and investigated quantitative CTA measures in all discordant cases.

\section{Materials and methods}

\section{Study population}

We included post-HTX adults who underwent routine ICA and coronary CTA for coronary wall imaging at our center for the exclusion (if CAV unknown) or for monitoring of CAV (if CAV known). The time interval between HTX and ICA was at least 10 months and no more than 7 days between ICA and CTA. Patients with acute allograft rejection, contraindications to contrast agents (e.g., severe renal dysfunction; blood creatinine $>1.5 \mathrm{mg} / \mathrm{dl}$ ), or those who did not undergo ICA and CTA were not included. The local ethics committee approved this retrospective observational cohort study (IRB\#442/18ek).

\section{CTA acquisition and coronary vessel segmentation}

All CTA scans were performed on a second-generation 128row dual-source CT scanner (Siemens Healthineers) using standard contrast-enhanced, ECG-gated/triggered protocols as described in detail in Supplemental Text S1. An expert reader (B.F.) with $>5$ years of experience in coronary CTA analyzed all coronary segments (luminal diameter $>1.5 \mathrm{~mm}$ ) following the 18-segment model (Society of Cardiac Computed Tomography; SCCT) [20] and using dedicated semi-automatic postprocessing software (QAngio, Research Edition, version 2.0.5; Medis Medical Imaging Systems). Left main, proximal right coronary artery, proximal left anterior descending, and proximal circumflex were considered proximal segments while the remaining as distal [21]. High reproducibility has been reported for this particular software package (wall burden intra-class correlation coefficient, 0.90 for intra- and 0.84 for inter-observer reliability $[12,19]$ ). (Supplemental Figure S1 shows the individual standard analytic steps as described elsewhere [11, 12, 19, 22].)

\section{Quantitative coronary CTA analysis}

First, we measured volume $\left(\mathrm{mm}^{3}\right)$ (lumen and wall) and length $(\mathrm{mm})$ of every coronary segment. Since long segments presented automatically with larger absolute volumes compared with short segments, we indexed all volumes by the length of the given segment. This step standardized the volumes and enabled comparison between segments with different lengths. All indexed volumes were treated as volumelength ratios (VLR; $\mathrm{mm}^{3} / \mathrm{mm}$ ) in all analyses. Analogous to plaque burden calculation in CHD [12, 22], we calculated wall burden (WB), defined as a proportion of coronary wall relative to the size of the entire segment (i.e., wall volume/wall + lumen volume; \%).

In a second step, we measured coronary wall composition using dynamic, tissue-specific attenuation thresholds as 
previously validated with IVUS [10-12, 23]. Hereby, four tissue types were assessed as absolute volumes $\left(\mathrm{mm}^{3}\right)$ : dense calcium, fibrotic, fibro-fatty, and low-attenuation tissue. To ensure comparability of segments of different sizes, we indexed all absolute values by the segmental wall volume and reported the composition as relative proportions (volume of individual proportion/wall volume; \%).

Image quality was evaluated according to the 2014 SCCT guidelines for interpretation and reporting of coronary CTA using a 4-point Likert scale ( 1 = excellent; 2 = good; $3=$ average; $4=$ poor) [20]. Segments with poor image quality were excluded from further analysis.

\section{Invasive coronary angiography}

All angiograms were acquired by experienced cardiologists using standard techniques according to the most recent guidelines and standard protocol in our clinic (Supplemental Text S2). An expert reader (M.S.) with $>6$ years of ICA experience interpreted all angiograms, was blinded to all CTA findings, and used the SCCT 18-segment model [20] to ensure comparability with CTA. All coronary lesions were classified visually according to stenosis grade and type as recommended by the American College of Cardiology/American Heart Association (ACC/AHA) Task Force guidelines (grade 0, 0 $24 \% ; 1,25-49 \% ; 2,50-74 \% ; 3,75-90 \%$ and $>90 \% ; 4,100 \%$ and type $\mathrm{A},<10 \mathrm{~mm}$ length, concentric; $\mathrm{B}, 10-20 \mathrm{~mm}$ length, eccentric; and C, $>20 \mathrm{~mm}$ length, excessive tortuosity) [24]. We classified CAV-positive segments as follows: no $C A V$, no stenosis or stenosis 1-24\%; mild CAV, stenosis grade $25-49 \%$, type $\mathrm{A}$ or $\mathrm{B} ; \geq$ moderate $C A V$, stenosis $\geq 50 \%$ and type $\mathrm{A}-\mathrm{C}$.

\section{Statistical analysis}

Continuous variables were expressed as mean \pm standard deviation (SD) or median (interquartile range; IQR), while categorical variables were listed as frequencies and percentages. To increase readability, we tabulated all values as mean \pm SD and restricted the use of the median (IQR) to highly skewed or ordinal-scaled data. Differences of CTA measures between $\mathrm{CAV}$-positive vs. CAV-negative patients were tested with the Wilcoxon rank-sum or Fisher's exact test, as appropriate. Associations of individual CTA measures with CAV by ICA were tested with univariate logistic regression. Subsequently, we rendered independent CTA variables using multivariate regression, including all variables which were associated with CAV in the univariate analyses $(p<0.05)$. In a supplemental analysis, we provided univariate and multivariate results adjusted for segment location (proximal vs. distal).

After building a multivariate logistic regression model, which included all CTA variables independently associated with $\mathrm{CAV}$, we calculated relative probability of having $\mathrm{CAV}$ in each coronary segment (continuous measure with range, $0-1$; equation provided in Supplemental Text S3). Using these probabilities, we computed areas under receiver operator curve (AUC/ROC) as measures of discriminatory capacity to detect CAV. Differences between AUC curves were tested with the likelihood-ratio test for nested models. In an explorative analysis, we estimated a threshold for the CTA-derived probability using the Youden index [25] (equal weight on sensitivity and specificity) including sensitivity and specificity for the given threshold.

Finally, we categorized the CTA-based probability into tertiles (i.e., low, intermediate, and high) and tabulated these against the CAV grades on ICA (none, mild, and $\geq$ moderate). We assessed the concordance between CTA and ICA and identified groups of segments with the highest concordance and discordance. Level of significance was defined as two-sided $p<0.05$, and Stata 15.0 was used for all statistical analyses.

\section{Results}

\section{Study population and evaluable coronary segments}

Among 42 (84\%) men and 8 (16\%) women (mean age of 53.6 \pm 11.9 years), who were included in the analytic cohort, CAV prevalence was $38 \%(19 / 50)$ on ICA. The mean interval between HTX and CTA was $6.7 \pm 4.7$ years, while the median interval between ICA and CTA was $1(0-1)$ day. Dilative cardiomyopathy $(58.0 \%)$ was the most frequent indication for HTX, and the most common comorbidities were renal dysfunction (44\%), dyslipidemia (26\%), and diabetes mellitus $(22 \%)$. Detailed demographics and medical treatment information are provided in Table 1 and Supplemental Table S1, respectively.

Table 1 Patient characteristics

\begin{tabular}{ll}
\hline Demographics & All $(n=50)$ \\
Age, years & $53.4 \pm 12.0$ \\
Male, $n(\%)$ & $42(84)$ \\
BMI, $\mathrm{kg} / \mathrm{m}^{2}$ & $27.0 \pm 3.9$ \\
Primary indication for HTX, $n(\%)$ & \\
Dilatative cardiomyopathy & $29(58)$ \\
Ischemic heart disease & $16(32)$ \\
Other & $5(10)$ \\
Cardiovascular risk factors, $n(\%)$ & \\
Diabetes & $11(22)$ \\
Art. hypertension & $6(12)$ \\
Hyperlipidemia & $13(26)$ \\
Atrial fibrillation & $4(8)$ \\
Peripheral artery disease & $2(4)$ \\
Renal disorder & $22(44)$ \\
Current smoker & $2(4)$ \\
\hline
\end{tabular}

$B M I$ body mass index 
Table 2 Quantitative CTA measures of coronary segments stratified by CAV status diagnosed on ICA

\begin{tabular}{lcccr}
\hline CTA measures & $\begin{array}{l}\text { All segments } \\
(n=632)\end{array}$ & $\begin{array}{l}\text { CAV-negative on ICA } \\
(n=591)\end{array}$ & $\begin{array}{l}\text { CAV-positive on ICA } \\
(n=41)\end{array}$ & $p$ \\
\hline Volume-length ratio, $\mathrm{mm}^{3} / \mathrm{mm}$ & & & & \\
$\quad$ Lumen & $5.8 \pm 4.0$ & $5.8 \pm 4.7$ & $5.9 \pm 5.0$ & 0.864 \\
$\quad$ Wall & $7.7 \pm 3.2$ & $7.5 \pm 2.9$ & $10.6 \pm 4.5$ & $<0.001$ \\
Wall burden, \% & $59.1 \pm 8.6$ & $59.5 \pm 8.4$ & $66.3 \pm 10.0$ & $<0.001$ \\
Composition, \% & & & & $<.2 \pm 6.1$ \\
$\quad$ Dense calcium & $1.0 \pm 2.8$ & $0.8 \pm 2.3$ & $48.7 \pm 11.4$ & 0.033 \\
Fibrotic tissue & $44.7 \pm 11.2$ & $44.4 \pm 11.1$ & $17.4 \pm 7.4$ & 0.204 \\
Fibro-fatty tissue & $18.6 \pm 7.8$ & $18.7 \pm 7.8$ & $7.1 \pm 6.1$ & 0.163 \\
Low-attenuation tissue & $8.5 \pm 6.6$ & $8.6 \pm 6.7$ & \\
\hline
\end{tabular}

$C A V$ cardiac allograft vasculopathy; $C T A$ computed tomography angiography; ICA invasive coronary angiography
Despite relatively high heart rate (mean $74.1 \pm$ $8.5 \mathrm{bpm}$ ), the median image quality was rated as good (IQR, excellent-good); (correlation of heart rate with image quality, $r=0.28 ; p=0.05$ ). Ultimately, 56 segments had poor image quality and were excluded from the analysis. Median dose length product and effective dose were 413.5 (225.0-678.0) $\mathrm{mGy} \mathrm{cm}$ and 5.8 (3.2-9.5) $\mathrm{mSv}$, respectively.

Overall, 692 coronary segments were evaluable on CTA, and among these, 632/692 (91.4\%) segments had a corresponding partner on ICA. Hence, the final analytic sample consisted of 632 coronary segments, of which 190/632 $(30.1 \%)$ and $442 / 632(69.9 \%)$ segments were classified as proximal and distal, respectively.

\section{Quantitative coronary CTA analysis}

On CTA, the mean wall and lumen VLRs were $7.7 \pm 3.2$ and $5.8 \pm 4.7 \mathrm{~mm}^{3} / \mathrm{mm}$, respectively, while the mean WB was $59.1 \pm 8.6 \%$ (Table 2 ). WB was higher in distal coronary segments $(62 \%$ vs. $56 \% ; p<0.001)$, while coronary lumen and wall VLRs were twofold lower compared with the proximal segments (lumen, $4.4 \pm 3.1$ vs. $9.5 \pm 6.0 \mathrm{~mm}^{3} / \mathrm{mm}$, and wall, $6.7 \pm 2.5$ vs. $10.0 \pm 3.3 \mathrm{~mm}^{3} / \mathrm{mm} ; p<0.001$ for both; Supplemental Table S2).

Regarding composition, coronary walls consisted of $44.7 \%$ fibrotic, $18.6 \%$ fibro-fatty, $8.5 \%$ low-attenuation, and $1.0 \%$ calcified tissue (Table 2), while the remaining $27.2 \%$ were outside of the tissue-specific thresholds (e.g., media border). Here is to be noted that the proportion of fibrotic tissue was significantly higher, while proportions of calcified, fibro-fatty, and low-attenuation tissue were significantly lower in the distal compared with that in proximal coronary segments $(p<0.001$ for all; Supplemental Table S2).

\section{Invasive coronary angiography}

On ICA, $19 / 50(38 \%)$ patients and 41/632 (6.5\%) coronary segments were rated as CAV-positive. Among patients with at least one coronary lesion, median $1(1-3)$ segments were classified as CAV-positive on ICA. Single-, double-, and triplevessel diseases were present in 12/19 (63\%), 4/19 (21\%), and $3 / 19(16 \%)$ patients, respectively. Median stenosis grade was 2 (2-2) (50-75\% luminal stenosis), while the most frequent lesion type was B $(25 / 49 ; 51 \%)$ followed by C $(18 / 49 ; 37 \%)$ and $\mathrm{A}(6 / 49 ; 12 \%)$.

\section{Quantitative coronary CTA measures in segments with and without CAV on ICA}

Coronary wall VLR was significantly larger in the 41 CAVpositive segments compared with those without CAV (10.6 \pm 4.5 vs. $\left.7.5 \pm 2.9 \mathrm{~mm}^{3} / \mathrm{mm} ; p<0.001\right)$. On the other hand, coronary lumen VLR did not differ significantly between CAV-positive and CAV-negative segments $(p=0.864)$. Accordingly, WB was higher in segments with CAV compared with those without CAV $(66.3 \pm 10.0$ vs. $59.5 \pm 8.4 \%$; $p<0.001$ ).

Regarding the wall composition, CAV-positive coronary segments had more fibrotic tissue $(48.7 \pm 11.4$ vs. $44.4 \pm$ $11.1 \% ; p=0.033)$ and dense calcium $(4.2 \pm 6.1$ vs. $0.8 \pm$ $2.3 \% ; p<0.001)$ compared with CAV-negative segments. Proportions of fibro-fatty and low-attenuation tissue did not differ between CAV-positive and CAV-negative segments ( $p=0.204$ and 0.163 , respectively) (Table 2 ).

In the univariate logistic regression, coronary wall VLR, $\mathrm{WB}$, and calcified and fibrotic tissue were associated with CAV $\left(\mathrm{OR}=1.04-1.28 ; p \leq 0.019\right.$ (ORs per $\mathrm{mm}^{3} / \mathrm{mm}$ or $\%$ increase as appropriate) (Table 3 ). In the multivariate analysis, coronary wall VLR, WB, and fibrotic tissue remained independently associated with $\mathrm{CAV}(\mathrm{OR}=1.27 ; p<0.001, \mathrm{OR}=$ 
Table 3 Association of CTA parameters with presence of CAV on ICA

\begin{tabular}{|c|c|c|c|c|c|c|}
\hline \multirow[t]{2}{*}{$n=632$ segments; CAV-positive $n=41$} & \multicolumn{3}{|c|}{ Univariate } & \multicolumn{3}{|c|}{ Multivariate* } \\
\hline & OR & $95 \% \mathrm{CI}$ & $p$ & OR & $95 \% \mathrm{CI}$ & $p$ \\
\hline \multicolumn{7}{|l|}{ Volume-length ratio (per $\mathrm{mm}^{3} / \mathrm{mm}$ ) } \\
\hline Lumen & 1.01 & $0.94-1.07$ & 0.876 & - & - & - \\
\hline Wall & 1.28 & $1.17-1.40$ & $<0.001$ & 1.27 & $1.14-1.42$ & $<0.001$ \\
\hline Wall burden (per \%) & 1.13 & $1.08-1.19$ & $<0.001$ & 1.09 & $1.05-1.14$ & $<0.001$ \\
\hline \multicolumn{7}{|l|}{ Composition, relative (per \%) } \\
\hline Dense calcium & 1.20 & $1.12-1.28$ & $<0.001$ & 1.08 & $1.00-1.18$ & 0.051 \\
\hline Fibrotic tissue & 1.04 & $1.01-1.07$ & 0.019 & 1.06 & $1.02-1.10$ & 0.002 \\
\hline Fibro-fatty tissue & 0.98 & $0.94-1.02$ & 0.313 & - & - & - \\
\hline Low-attenuation tissue & 0.96 & $0.91-1.01$ & 0.154 & - & - & - \\
\hline
\end{tabular}

Coronary wall VLR, wall burden and the proportion of dense calcium and fibrotic tissue were associated with CAV. Notably, the association of fibrotic tissue with CAV increased in the multivariate analysis, emphasizing its independence of the other factors. *Includes all variables $p<0.05$ in the univariate analysis. $C A V$, cardiac allograft vasculopathy; $C I$, confidence interval; $O R$, odds ratio

$1.09 ; p<0.001$, and $\mathrm{OR}=1.06 ; p=0.002$, respectively) (Table 3). All three CTA measures remained significantly associated with CAV after further adjustment for segment location $(p \leq 0.002$ for all; Supplemental Table S3).

Mean CTA-derived probability to have CAV was $6 \pm 11 \%$ (range $0-97 \%$ ) (derived from logistic model nesting coronary wall VLR, WB, and fibrotic tissue; Supplemental Text S3 provides the corresponding formula for calculation of individual combined probability). The probability reached a high discriminatory capacity for $\mathrm{CAV}(\mathrm{AUC}=0.83 ; 95 \% \mathrm{CI}$, 0.75-0.90) (Fig. 1). Even though the quantitative CTA did not focus on accessing luminal stenosis and, therefore, was not directly comparable with ICA, in a direct exploratory comparison of CTA vs. ICA, the sensitivity and specificity were relatively high ( $78 \%$ and $75 \%$, respectively, by using a threshold of $5 \%$ calculated by the Youden index).

\section{Concordance CTA vs. ICA}

Overall, there was a moderate concordance of $37 \%$ (233/632) between the coronary CTA and ICA (Table 4). As expected, the concordance was substantially higher in those with advanced disease (22/25 (88\%)) compared with those with none or mild disease on ICA (208/591 (37\%) and 3/16 (19\%), respectively). Focusing on the extremes, namely the difference of two categories, coronary CTA classified 177/632 (28\%) segments as a high probability for having CAV without a corresponding correlate on ICA. Not surprisingly, the discordant 177 segments had higher coronary wall VLR, WB, and more fibrotic tissue $(p<0.001$ for all) compared with segments with low and intermediate probability and no CAV on ICA. Figure 2c provides an example of a discordant case. Moreover, the discordant segments were more frequently distal segments $(112 / 177(63 \%)$ vs. $65 / 177(37 \%) ; p=0.012)$. On the other hand, only $1 / 632(0.2 \%)$ segment was classified as a low probability by CTA but presented with $\geq$ moderate CAV on ICA.

\section{Discussion}

In this study, we investigated the association of quantitative coronary CTA with CAV and explored the concordance between CTA and ICA. This study had two major findings: (1) CTA-derived coronary wall volume-length ratio, wall burden, and proportion of fibrotic tissue were independently associated with CAV, and (2) concordance between CTA and ICA was high in advanced CAV, while discordance was driven predominantly by distal coronary segments with changes of coronary wall on CTA but without CAV on ICA. Our study identified the CTA-derived coronary wall VLR, WB, and proportion of fibrotic tissue as independent measures of $\mathrm{CAV}$; corroborated the high performance of CTA to detect advanced CAV; and revealed a potential advantage of quantitative CTA in early stages of CAV.

Due to rapid technical progress, coronary CTA has become an alternative to ICA for the exclusion of CHD in patients with chest pain [13-15, 26, 27] and has received Ib recommendation in the most recent 2019 European Society of Cardiology guidelines for the diagnosis and management of chronic coronary syndromes [28]. However, coronary CTA has not become a routine procedure to detect CAV in HTX patients yet (class IIb recommendation [3]). In HTX patients, the primary concern is image quality. The limiting factor, influencing the image quality, is the frequently observed high heart rate and a limited response of HTX patients to heart rate modulation (e.g., beta-blockers).

While in older scanners image quality depended strongly on the heart rate [29-31], recent studies have shown that contemporary CTA technology reaches diagnostic image quality 


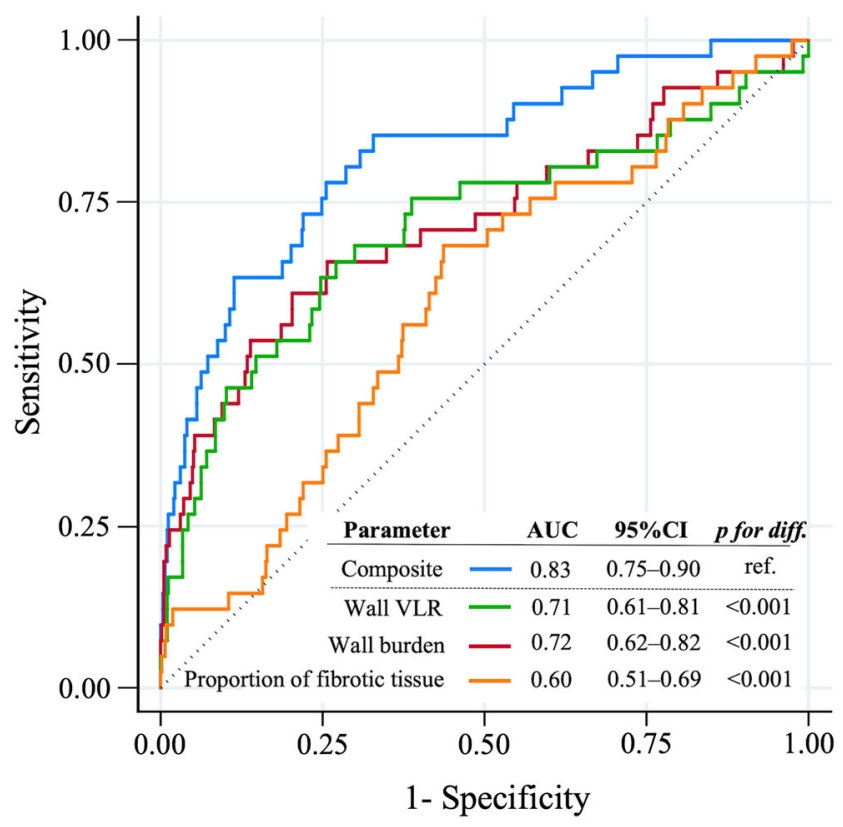

Fig. 1 Discriminatory capacity of quantitative CTA for the detection of CAV. Receiver operator characteristics curves for individual quantitative CTA measures (coronary wall volume-length ratio (VLR; $\mathrm{mm}^{3} / \mathrm{mm}$ ), wall burden (\%), and fibrotic tissue proportion (\%)) and the composite of all three parameters (i.e., logistic regression-based probability). The composite reached the highest discriminatory capacity. $p$ values indicate significantly larger AUC (0.83) compared with the AUCs of the individual CTA measures. VLR, volume-length ratio

in HTX patients with heart rates $>70 \mathrm{bpm}$ at reasonable radiation exposure (4.3-6.6 mSv) [18, 19, 32, 33]. Our study endorses these results by revealing good image quality at a mean heart rate of $74 \mathrm{bpm}$ and median effective radiation dose of $5.8 \mathrm{mSv}$. One could argue that the effective dose in CTA is higher than the reported average median effective dose of diagnostic ICA (3.3 mSv) [34]. Nevertheless, several recent studies using the most recent CTA technology have reported excellent images at effective dose of $0.3-2.9 \mathrm{mSv}$ despite heart rates above $70 \mathrm{bpm}$ or heart rhythm irregularities [35-37]. Therefore, further advances in temporal resolution and introduction of novel protocols, combining low $\mathrm{kVp}$ and iterative reconstruction algorithms, will likely lead to a further decline of radiation necessary for diagnostic image quality in HTX patients.

Considering the technical improvement of coronary CTA, it is not surprising that qualitative detection of obstructive coronary stenoses $(\geq 50 \%)$ on CTA agrees well with ICA not only in patients with CHD but also in HTX patients (negative predictive value, 98-100\%) [17, 27, 33, $38,39]$. Quantitative coronary wall analysis has become a supplementary tool to qualitative CTA by providing additional information about coronary wall volume and composition. In patients with CHD, quantitative CTA has revealed a good agreement with IVUS [11, 12] and has become a standard to monitor changes of atherosclerotic plaques in numerous studies using serial CTA [40-43]. Nevertheless, there is only limited evidence about the utilization of quantitative CTA in HTX patients. For instance, Karolyi et al used serial CTA to show that quantitative assessment of coronary walls is feasible to measure coronary wall changes over time and that progression of coronary wall volume is driven by noncalcified components [19]. Our study underlines the feasibility and enhances these results by identifying coronary wall VLR, WB, and proportion of fibrotic tissue as independent indicators of CAV.

Regarding pathophysiology of CAV, our results confirmed a series of histopathological studies which have shown that $\mathrm{CAV}$ is primarily driven by fibroproliferation [4-7], reflected in a higher proportion of fibrotic tissue in CAV-positive segments. Moreover, CAV has been reported to progress typically in a biphasic manner. Namely, CAV progression starts with an initial phase, including early intimal thickening with an expansion of the external elastic membrane and relative preservation of the lumen. The initial phase is then followed by an

Table 4 CTA vs. ICA for the detection of CAV

\begin{tabular}{|l|c|c|c|c|}
\hline & \multicolumn{3}{|c|}{ CAV by ICA } & \\
\hline CAV probability by CTA* & None & Mild & $\geq$ Moderate & Total \\
\hline T1: low (<2\%) & $208(32.9)$ & $2(0.3)$ & $1(0.2)$ & $211(33.3)$ \\
\hline T2: medium (2.0-4.5\%) & $206(32.6)$ & $3(0.5)$ & $2(0.3)$ & $211(33.3)$ \\
\hline T3: high (> 4.5\%) & $177(28.0)$ & $11(1.7)$ & $22(3.5)$ & $211(33.3)$ \\
\hline Total & $591(93.5)$ & $16(2.5)$ & $25(4.0)$ & $632(100.0)$ \\
\hline
\end{tabular}

The discordance between CTA and ICA was driven by segments which have shown large coronary wall changes on CTA but were not classified as CAVpositive by the ICA ( $n=177$; red lower left field). *Based on the logistic regression including coronary wall volume-length ratio, wall burden, and proportion of fibrotic tissue. $C A V=$ cardiac allograft vasculopathy; $C T A=$ computed tomography angiography; $I C A=$ invasive coronary angiography 
advanced phase with constrictive remodeling and luminal stenosis $[5,7]$. While ICA captures merely the coronary lumen, it may miss the early phases of CAV. The fact that CTA enables measurements of coronary walls regardless of luminal stenosis may explain why our sensitivity and specificity of quantitative CTA (78\% and 75\%, respectively) were lower than the usually reported values for qualitative CTA (assessment of significant stenosis ( $\geq 50 \%$ ); $94 \%$ and $92 \%$, respectively) [33].

The reason becomes even more apparent if investigating the concordance between quantitative CTA and ICA. We found a high concordance (88\%) between both methods in advanced CAV, which confirms that lesions with coronary wall changes and lumen narrowing are detectable by both methods accurately. These results are in accord with a metaanalysis by Wever-Pinzon et al, who investigated the accuracy of qualitative CTA to detect CAV in comparison with ICA [33]. However, the discordance was driven by segments which were classified as CAV-positive by CTA and CAVnegative by ICA (i.e., segments with coronary wall changes but without a luminal stenosis; example, Fig. 2c). In fact, quantitative CTA has shown significant coronary wall changes in $28 \%$ of segments rated as normal on ICA (Tab. 4). Vice versa, CTA rated only one $(0.2 \%)$ coronary segment as a low probability of having CAV while the ICA reported advanced $\mathrm{CAV}$. These findings are striking and suggest a higher sensitivity and a higher negative predictive value of quantitative CTA for the detection of early CAV compared with ICA.
Future prospective studies with less mechanistic and more prognostic endpoints (e.g., survival) are needed to confirm this hypothesis.

Moreover, the discordance between CTA and ICA was the highest in distal coronary segments, those not always accessible by IVUS, underlining the importance to evaluate the entire coronary tree, representing a clear advantage of CTA compared with IVUS. Indexing of segmental volumes by segmental length (i.e., VLR) and treating coronary wall components as proportions standardized the values and permitted comparison of segments at different locations. In a supplemental analysis, we showed that segmental VLR, WB, and proportion of fibrotic tissue are all independently associated with CAV even after adjustment for segment location. The independence of segment location further emphasizes the robustness and generalizability of the quantitative CTA measures.

In general, coronary walls and lumen were smaller, while WB and proportion of fibrotic tissue were higher in the distal coronary segments compared with those in the proximal segments. In other words, in the course of the vessel, the lumen became smaller while the wall did not get necessarily thinner. This observation should get further attention in future studies to investigate whether an increasing WB towards distal coronary segments reflects normal anatomy or also a sign of CAV.

Potential clinical implications of our results may include earlier detection of CAV leading to a sooner initiation or more aggressive regimen of medical therapy, intensification of
Fig. 2 Imaging examples stratified by CAV status. Left coronary arteries of three patients with (a) CAV on ICA and CTA, corresponding luminal stenoses on ICA and wall thickening on CTA; (b) patient without signs of $\mathrm{CAV}$, with normal coronary lumen on ICA and thin coronary wall on CTA; and (c) patient with normal coronary lumen on ICA (also no stenosis on CTA), but wall thickening (red arrowheads) on CTA. Red dotted lines mark the reference areas of interest. CAV, cardiac allograft vasculopathy; CTA, computed tomography angiography; ICA, invasive coronary angiography

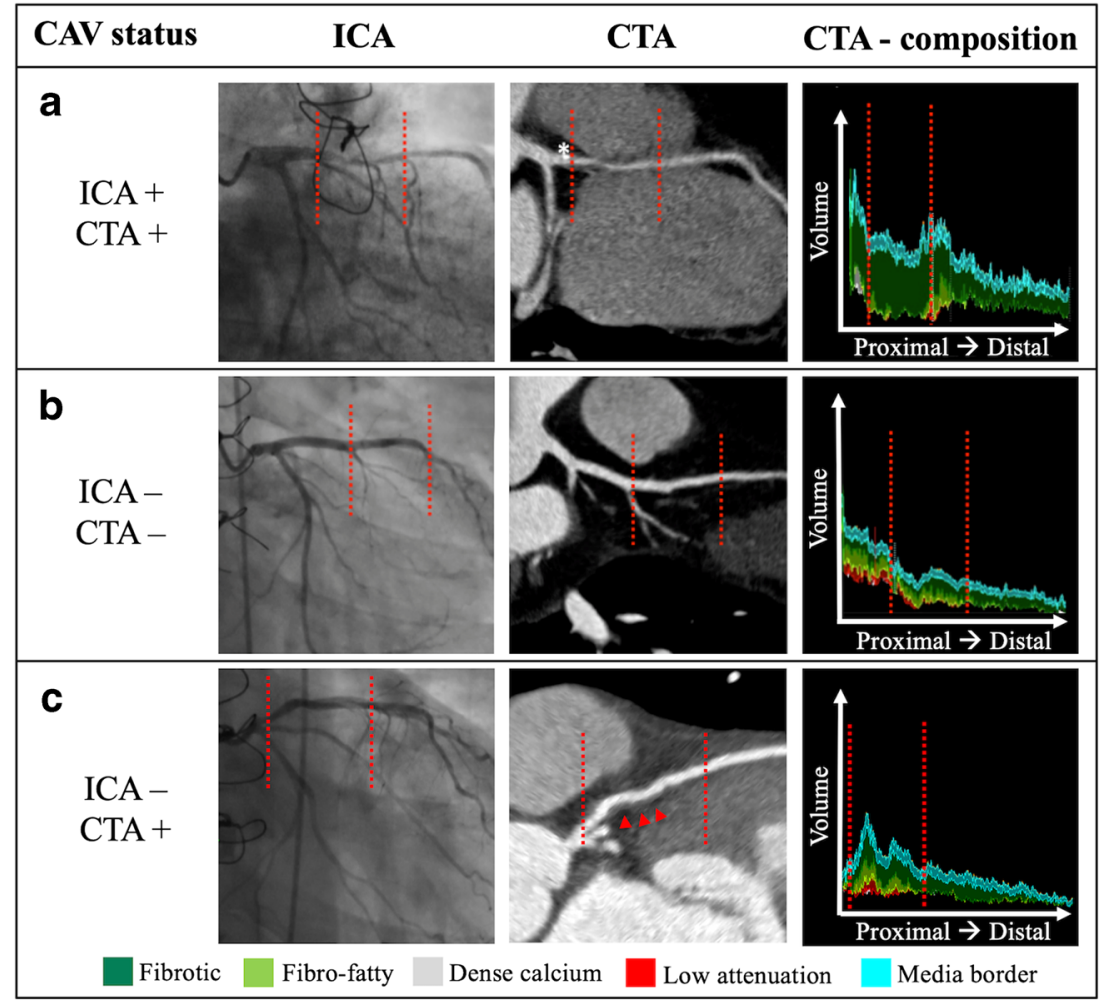


follow-up visits, and more intensive management of risk factors. Notably, in our cohort, $>80 \%$ of patients were on NSAR and statins, and around $60 \%$ received tacrolimus/everolimus, which seem to be the most effective treatment/prevention strategy for CAV. While the long-term efficacy of these lastnamed medications remains unclear [44], quantitative CTA may represent a novel tool to monitor not only the progression of CAV but also the effects of immunosuppressive drugs. In fact, quantitative coronary CTA has been successfully utilized for monitoring of coronary plaques in patients receiving statins $[40,45,46]$ in contrast to earlier studies, such as the SATURN trial [47], which traditionally relayed on IVUS.

We acknowledge several limitations of this retrospective single-center observational cohort study. First, we included only HTX patients and did not compare the CTA results with general population. Thus, future studies, preferably in individuals without known CHD, should define normal values of VLR, WB, and fibrotic tissue. We did not have information about the cardiovascular risk profile of our heart donors, and preexisting CHD may have influenced our measurements (e.g., calcifications). Serial approaches, which would include a baseline CTA exam and would focus on changes of the qualitative CTA measures during follow-up, may provide more insights into the pathogenesis of CAV. Moreover, the sample size in our study was relatively small. Future larger studies should include the evaluation of clinical outcomes and investigate the incremental value of quantitative over qualitative CTA.

\section{Conclusion}

Coronary CTA-derived wall VLR, WB, and proportion of fibrotic tissue were independently associated with CAV on ICA. While the concordance between CTA and ICA was high in advanced CAV, the discordance was mainly driven by distal coronary segments showing changes of coronary walls on CTA but without corresponding stenosis on ICA. These segments may represent those with non-stenotic coronary wall changes, related to early stages of CAV. Thus, quantitative CTA, in particular the combination of VLR, WB, and proportion of fibrotic tissue, may aid the detection of early CAV, guide medical treatment, and ultimately improve patients' outcomes.

Funding information Open Access funding provided by Projekt DEAL.

\section{Compliance with ethical standards}

Guarantor The scientific guarantor of this publication is Lukas Lehmkuhl, MD.

Conflict of interest The authors of this manuscript declare no relationships with any companies whose products or services may be related to the subject matter of the article.
Statistics and biometry One of the authors has significant statistical expertise.

No complex statistical methods were necessary for this paper.

Informed consent Written informed consent was waived by the Institutional Review Board.

Ethical approval Institutional Review Board approval was obtained.

Methodology

- Retrospective

- Observational

- Performed at one institution

Open Access This article is licensed under a Creative Commons Attribution 4.0 International License, which permits use, sharing, adaptation, distribution and reproduction in any medium or format, as long as you give appropriate credit to the original author(s) and the source, provide a link to the Creative Commons licence, and indicate if changes were made. The images or other third party material in this article are included in the article's Creative Commons licence, unless indicated otherwise in a credit line to the material. If material is not included in the article's Creative Commons licence and your intended use is not permitted by statutory regulation or exceeds the permitted use, you will need to obtain permission directly from the copyright holder. To view a copy of this licence, visit http://creativecommons.org/licenses/by/4.0/.

\section{References}

1. Lund LH, Edwards LB, Kucheryavaya AY et al (2015) The registry of the International Society for Heart and Lung Transplantation: thirty-second official adult heart transplantation report-2015; focus theme: early graft failure. J Heart Lung Transplant 34:1244 1254. https://doi.org/10.1016/j.healun.2015.08.003

2. Badano LP, Miglioranza MH, Edvardsen T et al (2015) European Association of Cardiovascular Imaging/Cardiovascular Imaging Department of the Brazilian Society of Cardiology recommendations for the use of cardiac imaging to assess and follow patients after heart transplantation. Eur Heart J Cardiovasc Imaging 16:919948. https://doi.org/10.1093/ehjci/jev139

3. Costanzo MR, Dipchand A, Starling R et al (2010) The International Society of Heart and Lung Transplantation Guidelines for the care of heart transplant recipients. J Heart Lung Transplant 29:914-956. https://doi.org/10.1016/j.healun. 2010.05.034

4. Chih S, Chong AY, Mielniczuk LM, Bhatt DL, Beanlands RS (2016) Allograft vasculopathy: the Achilles' heel of heart transplantation. J Am Coll Cardiol 68:80-91. https://doi.org/10.1016/j.jacc. 2016.04.033

5. Hiemann NE, Wellnhofer E, Knosalla C et al (2007) Prognostic impact of microvasculopathy on survival after heart transplantation: evidence from 9713 endomyocardial biopsies. Circulation 116: 1274-1282. https://doi.org/10.1161/CIRCULATIONAHA.106. 647149

6. Torres HJ, Merello L, Ramos SA et al (2011) Prevalence of cardiac allograft vasculopathy assessed with coronary angiography versus coronary vascular ultrasound and virtual histology. Transplant Proc 43:2318-2321. https://doi.org/10.1016/j.transproceed.2011.06.002

7. Tsutsui H, Ziada KM, Schoenhagen P et al (2001) Lumen loss in transplant coronary artery disease is a biphasic process involving early intimal thickening and late constrictive remodeling. Circulation 104:653-657. https://doi.org/10.1161/hc3101.093867 
8. Javaheri A, Saha N, Lilly SM (2016) How to approach the assessment of cardiac allograft vasculopathy in the modern era: review of invasive imaging modalities. Curr Heart Fail Rep. https://oi.org/ 10.1007/s11897-016-0283-y

9. St Goar FG, Pinto FJ, Alderman EL et al (1992) Intracoronary ultrasound in cardiac transplant recipients. In vivo evidence of "angiographically silent" intimal thickening. Circulation 85:979-987

10. Boogers MJ, Broersen A, van Velzen JE et al (2012) Automated quantification of coronary plaque with computed tomography: comparison with intravascular ultrasound using a dedicated registration algorithm for fusion-based quantification. Eur Heart J 33: 1007-1016. https://doi.org/10.1093/eurheartj/ehr465

11. de Graaf MA, Broersen A, Kitslaar PH et al (2013) Automatic quantification and characterization of coronary atherosclerosis with computed tomography coronary angiography: cross-correlation with intravascular ultrasound virtual histology. Int J Cardiovasc Imaging 29:1177-1190. https://doi.org/10.1007/s10554-013-0194$\mathrm{x}$

12. Park H-B, Lee BK, Shin S et al (2015) Clinical feasibility of 3D automated coronary atherosclerotic plaque quantification algorithm on coronary computed tomography angiography: comparison with intravascular ultrasound. Eur Radiol 25:3073-3083. https://doi.org/ 10.1007/s00330-015-3698-Z

13. Hoffmann U, Truong QA, Schoenfeld DA et al (2012) Coronary $\mathrm{CT}$ angiography versus standard evaluation in acute chest pain. $\mathrm{N}$ Engl J Med 367:299-308. https://doi.org/10.1056/ NEJMoa1201161

14. Douglas PS, Hoffmann U, Patel MR et al (2015) Outcomes of anatomical versus functional testing for coronary artery disease. $\mathrm{N}$ Engl J Med 372:1291-1300. https://doi.org/10.1056/ NEJMoa1415516

15. SCOT-HEART investigators (2015) CT coronary angiography in patients with suspected angina due to coronary heart disease (SCOT-HEART): an open-label, parallel-group, multicentre trial. Lancet 385:2383-2391. https://doi.org/10.1016/S0140-6736(15) 60291-4

16. Hoffmann U, Bamberg F, Chae CU et al (2009) Coronary computed tomography angiography for early triage of patients with acute chest pain: the ROMICAT (rule out myocardial infarction using computer assisted tomography) trial. J Am Coll Cardiol 53:16421650. https://doi.org/10.1016/j.jacc.2009.01.052

17. Mittal TK, Panicker MG, Mitchell AG, Banner NR (2013) Cardiac allograft vasculopathy after heart transplantation: electrocardiographically gated cardiac CT angiography for assessment. Radiology 268:374-381. https://doi.org/10.1148/radiol.13121440

18. Bartykowszki A, Kolossváry M, Jermendy ÁL et al (2017) Image quality of prospectively ECG-triggered coronary CT angiography in heart transplant recipients. AJR Am J Roentgenol 210:314-319. https://doi.org/10.2214/AJR.17.18546

19. Károlyi M, Kolossváry M, Bartykowszki A et al (2018) Quantitative CT assessment identifies more heart transplanted patients with progressive coronary wall thickening than standard clinical read. J Cardiovasc Comput Tomogr. https://doi.org/10.1016/j. ject.2018.11.006

20. Leipsic J, Abbara S, Achenbach S et al (2014) SCCT guidelines for the interpretation and reporting of coronary $\mathrm{CT}$ angiography: a report of the Society of Cardiovascular Computed Tomography Guidelines Committee. J Cardiovasc Comput Tomogr 8:342-358. https://doi.org/10.1016/j.jcct.2014.07.003

21. Kwan AC, May HT, Cater G et al (2014) Coronary artery plaque volume and obesity in patients with diabetes: the factor-64 study. Radiology 272:690-699. https://doi.org/10.1148/radiol.14140611

22. Rodriguez K, Kwan AC, Lai S et al (2015) Coronary plaque burden at coronary CT angiography in asymptomatic men and women. Radiology 277:73-80. https://doi.org/10.1148/radiol.2015142551
23. Heo R, Park H-B, Lee BK et al (2015) Optimal boundary detection method and window settings for coronary atherosclerotic plaque volume analysis in coronary computed tomography angiography: comparison with intravascular ultrasound. Eur Radiol. https://doi. org $/ 10.1007 / \mathrm{s} 00330-015-4121-5$

24. Ryan TJ, Faxon DP, Gunnar RM et al (1988) Guidelines for percutaneous transluminal coronary angioplasty. A report of the American College of Cardiology/American Heart Association Task Force on assessment of diagnostic and therapeutic cardiovascular procedures (subcommittee on percutaneous transluminal coronary angioplasty). Circulation 78:486-502

25. Youden WJ (1950) Index for rating diagnostic tests. Cancer 3:3235. https://doi.org/10.1002/1097-0142(1950)3:1<32::AIDCNCR2820030106>3.0.CO;2-3

26. NICE Guidelines (2010) Recent-onset chest pain of suspected cardiac origin: assessment and diagnosis. Available via https://www. nice.org.uk/guidance/cg95. Accessed 19 Apr 2017

27. Budoff MJ, Dowe D, Jollis JG et al (2008) Diagnostic performance of 64-multidetector row coronary computed tomographic angiography for evaluation of coronary artery stenosis in individuals without known coronary artery disease: results from the prospective multicenter ACCURACY (assessment by coronary computed tomographic angiography of individuals undergoing invasive coronary angiography) trial. J Am Coll Cardiol 52:1724-1732. https://doi. org/10.1016/j.jacc.2008.07.031

28. Knuuti J, Wijns W, Saraste A et al (2019) ESC Guidelines for the diagnosis and management of chronic coronary syndromes. Eur Heart J. https://doi.org/10.1093/eurheartj/ehz425

29. Raff GL, Gallagher MJ, O’Neill WW, Goldstein JA (2005) Diagnostic accuracy of noninvasive coronary angiography using 64-slice spiral computed tomography. J Am Coll Cardiol 46:552557. https://doi.org/10.1016/j.jacc.2005.05.056

30. Schroeder S, Kopp AF, Kuettner A et al (2002) Influence of heart rate on vessel visibility in noninvasive coronary angiography using new multislice computed tomography: experience in 94 patients. Clin Imaging 26:106-111. https://doi.org/10.1016/S08997071(01)00371-0

31. Latif M, Sanchez F, Sayegh K et al (2016) Volumetric single-beat coronary computed tomography angiography. J Comput Assist Tomogr 40:763-772. https://doi.org/10.1097/RCT. 0000000000000428

32. Rohnean A, Houyel L, Sigal-Cinqualbre A, To NT, Elfassy E, Paul JF (2011) Heart transplant patient outcomes: 5-year mean follow-up by coronary computed tomography angiography. Transplantation 91:583-588. https://doi.org/10.1097/TP.0b013e3182088b96

33. Wever-Pinzon O, Romero J, Kelesidis I et al (2014) Coronary computed tomography angiography for the detection of cardiac allograft vasculopathy: a meta-analysis of prospective trials. J Am Coll Cardiol 63:1992-2004. https://doi.org/10.1016/j.jacc.2014.01.071

34. Smith IR, Rivers JT (2008) Measures of radiation exposure in cardiac imaging and the impact of case complexity. Heart Lung Circ 17:224-231. https://doi.org/10.1016/j.hlc.2007.10.004

35. Feng R, Mao J, Liu X, Zhao Y, Tong J, Zhang L (2018) High-pitch coronary computed tomographic angiography using the thirdgeneration dual-source computed tomography: initial experience in patients with high heart rate. J Comput Assist Tomogr 42:248. https://doi.org/10.1097/RCT.0000000000000678

36. Andreini D, Mushtaq S, Pontone G et al (2018) Diagnostic performance of coronary $\mathrm{CT}$ angiography carried out with a novel wholeheart coverage high-definition CT scanner in patients with high heart rate. Int J Cardiol 257:325-331. https://doi.org/10.1016/j. ijcard.2017.10.084

37. Matveeva A, Schmitt RR, Edtinger K et al (2018) Coronary CT angiography in patients with atrial fibrillation: standard-dose and low-dose imaging with a high-resolution whole-heart CT scanner. 
Eur Radiol 28:3432-3440. https://doi.org/10.1007/s00330-0175282-1

38. Günther A, Aaberge L, Abildgaard A et al (2018) Coronary computed tomography in heart transplant patients: detection of significant stenosis and cardiac allograft vasculopathy, image quality, and radiation dose. Acta Radiol 59:1066-1073. https://doi.org/10.1177/ 0284185117748354

39. Miller JM, Rochitte CE, Dewey M et al (2008) Diagnostic performance of coronary angiography by 64-row CT. N Engl J Med 359: 2324-2336. https://doi.org/10.1056/NEJMoa0806576

40. Lo J, Lu MT, Ihenachor EJ et al (2015) Effects of statin therapy on coronary artery plaque volume and high-risk plaque morphology in HIV-infected patients with subclinical atherosclerosis: a randomised, double-blind, placebo-controlled trial. Lancet HIV 2: e52-e63. https://doi.org/10.1016/S2352-3018(14)00032-0

41. Foldyna B, Fourman LT, Lu MT et al (2018) Sex differences in subclinical coronary atherosclerotic plaque among individuals with HIV on antiretroviral therapy. J Acquir Immune Defic Syndr 78: 421-428 https://doi.org/10.1097/QAI.0000000000001686

42. Shin S, Park H-B, Chang H-J et al (2017) Impact of intensive LDL cholesterol lowering on coronary artery atherosclerosis progression: a serial CT angiography study. JACC Cardiovasc Imaging 10:437446. https://doi.org/10.1016/j.jcmg.2016.04.013

43. Li Z, Hou Z, Yin W et al (2016) Effects of statin therapy on progression of mild noncalcified coronary plaque assessed by serial coronary computed tomography angiography: a multicenter prospective study. Am Heart J 180:29-38. https://doi.org/10.1016/j. ahj.2016.06.023

44. Langstraat M, Musters KJS, Manintveld O, Masetti M, Potena L (2018) Coronary artery disease in heart transplantation: new concepts for an old disease. Transpl Int 31:787-827. https://doi.org/10. $1111 /$ tri. 13141

45. Foldyna B, Lo J, Mayrhofer T, Grinspoon SK, Hoffmann U, Lu MT (2019) Individual coronary plaque changes on serial CT angiography: within-patient heterogeneity, natural history, and statin effects in HIV. J Cardiovasc Comput Tomogr. https://doi.org/10.1016/j. ject.2019.08.011

46. Lee S-E, Chang H-J, Sung JM et al (2018) Effects of statins on coronary atherosclerotic plaques: the PARADIGM study. JACC Cardiovasc Imaging 11:1475-1484. https://doi.org/10.1016/j. jcmg.2018.04.015

47. Puri R, Nicholls SJ, Shao M et al (2015) Impact of statins on serial coronary calcification during atheroma progression and regression. J Am Coll Cardiol 65:1273-1282. https://doi.org/10.1016/j.jacc. 2015.01.036

Publisher's note Springer Nature remains neutral with regard to jurisdictional claims in published maps and institutional affiliations. 